\title{
Peritoneal Tuberculosis: Looking beyond the Typical Pathology
}

\author{
O’Dene Lewis*, Suneel Tammana, Peter Sealy \\ Department of Medicine, Howard University Hospital, Washington DC, USA \\ Email: odene.lewis@howard.edu, stammana@howard.edu, psealy@howard.edu
}

Received 8 December 2013; revised 8 January 2014; accepted 15 January 2014

Copyright (C) 2014 by authors and Scientific Research Publishing Inc.

This work is licensed under the Creative Commons Attribution International License (CC BY). http://creativecommons.org/licenses/by/4.0/

(c) (i) Open Access

\begin{abstract}
Peritoneal tuberculosis (TbP), an uncommon peritoneal infection, is commonly diagnosed in immigrants from developing countries and represents a substantial proportion of cases of extra pulmonary tuberculosis. The variability in patient presentation and the indolent nature of the infection, combined with limited diagnostic strategies available for TbP, often results in delayed diagnosis. Case: Described herein is the case of a 39 years old male recent immigrant from Mali (West Africa), with no significant medical history that presented to hospital with a four-day history of abdominal pain and swelling. Examination was significant for distended abdomen and shifting dullness. No signs and symptoms suggested pulmonary infection, however, QuantiFERON-TB Gold and purified protein derivative (PPD) test were positive suggesting latent Tb infection. In the absence of pulmonary tuberculosis, a diagnosis of TbP should be established histologically. Laparoscopic biopsy showed granuloma but the typical caseating granuloma of TbP was not seen. Nonetheless, based on the extent of the clinical and laboratory findings, the patient was diagnosed with TbP and anti Tb treatment ensued with successful outcome. Conclusion: The lack of caseating granulomas in the pathology should not rule out a diagnosis of TbP, especially in cases where accompanying evidence suggests some form of Tuberculosis.
\end{abstract}

\section{Keywords}

Peritoneal Tuberculosis; Tuberculosis; Tuberculosis Peritonitis

\section{Introduction}

Tuberculosis ( $\mathrm{Tb})$ infection caused by mycobacterium tuberculosis or other mycobacterium species is a major communicable disease worldwide. The infection is characterized by the formation of tuberculous granulomas

${ }^{*}$ Corresponding author. 
and caseous necrosis in tissue. Primary Tb infection occurs in the lung, the portal through which infection spreads to other sites such as the kidney, spine, genitals and the peritoneum [1].

TbP occurs in $4 \%-10 \%$ of patients with extra pulmonary tuberculosis [2]. Clinically active TbP can occur through hematogenous spread from active pulmonary lesions, rupture of infected caseous abdominal lymph nodes or reactivation of latent peritoneal foci. Direct spread may also occur from an initial focus in the intestine, fallopian tube or from an abscess that abuts the peritoneal cavity [3].

TbP can be asymptomatic or result in non-specific symptoms such as weight loss, abdominal pain, fever, abdominal distention, vomiting, diarrhea and anorexia. Early diagnosis of TbP is challenging owing to its protean clinical manifestations and the difficulty in obtaining specimens for tissue culture. Definitive diagnosis is usually established by histological biopsy assessment showing caseating granuloma, positive acid-fast bacillus, culture for Mycobacterium Tb or positive polymerase chain reaction [4]. Whereas a histological finding of caseating granuloma is considered required for definitive diagnosis of TbP, the literature documents the occurrence of non-caseating granulomas in peritoneal $\mathrm{Tb}[5]$.

TbP is still a medical concern in developing countries but is relatively uncommon in developed countries. However, due to increases of international travel and the frequency of immune suppressive diseases such as acquired immune deficiency syndrome (AIDS), physicians worldwide should be aware of TbP. Unless a high degree of suspicion is maintained, the diagnosis can easily be missed or delayed, resulting in increased morbidity and mortality [6].

\section{Case Report}

A 39 years old African American male with no significant past medical history presented to the Emergency Care Area (ECA) with a 4-day history of abdominal pain and swelling. These complaints were associated with fever (subjective), chills, decreased appetite and increase in abdominal girth. He had no history of hepatitis, yellow discoloration of his eyes or exposure to rodents. Review of systems was positive for constipation. Of significance he moved to the US from Mali in West Africa three years ago and his father has a history of being treated for Pulmonary Tuberculosis.

His examination was significant for abdominal distention with mild generalized tenderness and shifting dullness. No masses or organomegally was appreciated and his digital rectal examination was normal

Initial laboratory investigations were unremarkable. CT scan of the abdomen with contrast was performed (Figure 1). At that point the etiology of his ascites was unclear. Differentials included infectious, inflammatory or malignant causes. Paracentesis, QuantiFERON gold and PPD were done as well as other clinical investigations to rule out these causes (Table 1). QuantiFERON Gold test was positive ( $>1.31$ IU/ML). Purified Protein Derivative (PPD) was positive with induration at $10 \mathrm{~mm}$. Paracentesis was performed and $2 \mathrm{~L}$ of serous fluid was removed. Laboratory analysis showed a cloudy yellow fluid with 288 white blood cells (WBC), 1872 red blood cells (RBC) and 2 polymorphonuclear cells. Gram stain was negative. Special stains showed no tuberculli. Adenosine Deaminase (ADA) was positive at $93.3 \mathrm{U} / \mathrm{L}$ (ref range for $\mathrm{Tb}>92.1 \mathrm{U} / \mathrm{L}$ ). Bacterial, $\mathrm{Tb}$ (after six weeks) and fungal cultures were negative. Serum ascetic albumin gradient was less than $1.1 \mathrm{~g} / \mathrm{dl}(0.2 \mathrm{~g} / \mathrm{dl})$. Cytology showed no malignant cells and numerous chronic inflammatory cells and macrophages.

Clinical data was suggestive of peritoneal Tb but no overwhelming evidence. Laparoscopic biopsy was done to confirm the diagnosis of TbP. Operative findings included severe adhesions to the anterior abdominal wall impeding visualization during the operation. Histopathologic examination of the biopsy showed granulomatous inflammation without caseating necrosis (Figure 2).

Based on the clinical findings, labs and pathological assessment a decision was made for a trial of anti Tb drugs. Treatment included 1) Isoniazid $300 \mathrm{mg}$ once daily, 2) Rifampin $600 \mathrm{mg}$ once daily, 3) Ethambutol 800 mg once daily, 4) pyrazinamide $1000 \mathrm{mg}$ once daily. He was also started on B6 once daily. The patient responded well to therapy. He remained afebrile and regained his appetite. Subsequent follow up revealed that his abdominal pain and distention had improved. Therapy was recommended for a total of 9 months.

\section{Discussion}

The clinical manifestations of TbP are variable and it may mimic many diseases and conditions such as pyrexia of unknown origin, peritoneal carcinomatosis, abdominal lymphoma, pelvic mass, bacterial peritonitis and 


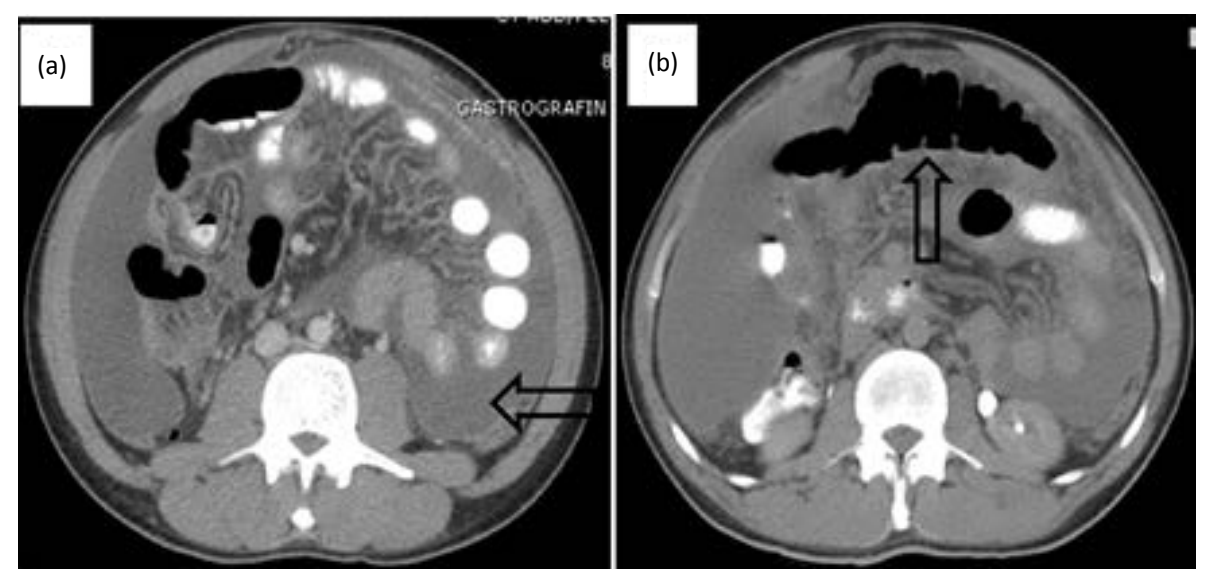

Figure 1. Computed Tomography of the abdomen. (a) CT scan shows large ascities (arrow) which displaces the small intestines and mesentery. (b) Probable inflammatory changes in the omentum (arrow) and in the mesentery of the rectosigmoid colon. Minimal bibasilar atelectasis, small left pleural effusion and small left cardiophrenic angle adenopathy are also present.

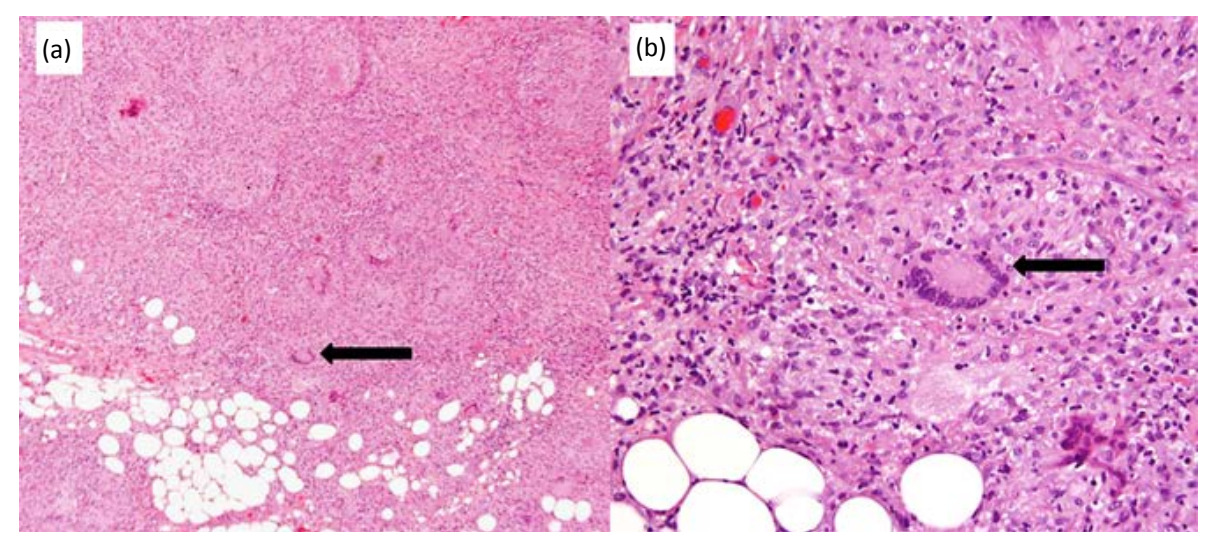

Figure 2. Histopathological findings of the omentum biopsy. Granulomatous inflammation (arrows) without caseating necrosis, indicated by epitheloid histiocytes at various magnifications (a) Low power $(\times 4)$ (b) High power $(\times 20)$.

non-responsive ascites of portal hypertension or cardiac failure origin [2].

Multiple case series, describing TbP infection, revealed the following common symptoms at presentation: abdominal swelling (65\% - 100\%), fever (54\% - 100\%), abdominal pain (36\% - 93\%), weight loss (37\% - 87\%), and diarrhea $(9 \%-27 \%)$. The physical findings included ascites $(51 \%-100 \%)$ and abdominal tenderness $(65 \%$ - 87\%) [7] [8].

In addition to symptomatology, lab test are used to guide in diagnosis of TbP. However, laboratory tests are nonspecific and abnormalities are related to chronic inflammation. Ascitic fluid analysis can be helpful in ascertaining a diagnosis and is usually yellow citrine but can take a cloudy, chylous or hematic appearance [9]. The ascitic fluid is often primarily composed of lymphocytes $(>60 \%)$ [10], however, neutrophils may predominate in cases of renal failure or infection with another microbial organism. Whereas no biochemical marker is specific for the diagnosis of TbP, a serum to ascetic albumin gradient (SAAG) less than $1.1 \mathrm{~g} / \mathrm{dl}$ is seen in $100 \%$ of patients with TbP [10]. However, SAAG along with the ascetic fluid assays of lactate dehydrogenase (LDH) and glucose have specificity too low to be recommended for the diagnosis of TbP [10]. Direct smear of the ascitic fluid with Ziehl-Nelson stain and cultures of ascetic fluid have a low diagnostic yield with a reported sensitivity and specificity of $0 \%-6 \%$ and less than $20 \%$ respectively [7]. In the case described above, the SAAG was less than $1.1 \mathrm{~g} / \mathrm{dl}$ but the stains and cultures of the ascetic fluid were negative.

Despite being nonspecific, increased serum and ascitic CA-125 levels have been observed in the majority of patients with $\mathrm{TbP}$ [9]. Anti $\mathrm{Tb}$ treatment has been shown to produce rapid falls in CA-125 levels paralleling 
Table 1. Laboratory data on admission to Howard University Hospital.

\begin{tabular}{|c|c|c|c|}
\hline Test/Analyte & Results & Test/Analyte & Results \\
\hline White blood cells & 5300/ul (3200 - 10,600) ul & HBsAG & Negative \\
\hline Hemoglobin & $14.4 \mathrm{~g} / \mathrm{dl}(12.1$ - 15.9) g/dl & Anti-HBs & Positive \\
\hline Platelets & $447 \times 10^{9} / \mathrm{L}(177-406) \times 10^{9} / \mathrm{L}$ & Anti-HBc & Positive \\
\hline $\begin{array}{l}\text { INR } \\
\text { PT }\end{array}$ & $\begin{array}{c}1.11(1.12-1.46) \text { INR } \\
13.8(12.5-14.5) \mathrm{sec}\end{array}$ & IgM-HBc & Negative \\
\hline PTT & $32.5(24.0-34.0) \mathrm{sec}$ & Anti-HCV & Negative \\
\hline Blood Urea Nitrogen & $11 \mathrm{mg} / \mathrm{dl}(7-25) \mathrm{mg} / \mathrm{dl}$ & HIV ELISA & Negative \\
\hline Creatinine & $1.0 \mathrm{mg} / \mathrm{dl}(0.7-1.4) \mathrm{mg} / \mathrm{dl}$ & C3 & $153 \mathrm{mg} / \mathrm{dl}(83$ - 177) mg/dl \\
\hline Total Bilirubin & $1.0 \mathrm{mg} / \mathrm{dl}(0.2-1.2) \mathrm{mg} / \mathrm{dl}$ & $\mathrm{C} 4$ & 33 mg/dl (15 - 45) mg/dl \\
\hline Total protein & $7.6 \mathrm{~g} / \mathrm{dl}(6.2-8.3) \mathrm{g} / \mathrm{dl}$ & ANA & Negative \\
\hline Albumin & $3.1 \mathrm{~g} / \mathrm{dl}(3.2-5.5) \mathrm{g} / \mathrm{dl}$ & $\mathrm{RF}$ & Negative \\
\hline AST & $48 \mathrm{mu} / \mathrm{ml}(0-50) \mathrm{mu} / \mathrm{ml}$ & Anti-SMA & Negative \\
\hline ALT & $38 \mathrm{mu} / \mathrm{ml}(0-55) \mathrm{mu} / \mathrm{ml}$ & TSH & $2.90 \mathrm{mu} / \mathrm{ml}(0.4-4.0) \mathrm{mu} / \mathrm{ml}$ \\
\hline Alk Phos & $72 \mathrm{mu} / \mathrm{ml}(30-165) \mathrm{mu} / \mathrm{ml}$ & CEA & Negative \\
\hline Amylase & $106 \mathrm{u} / \mathrm{l}(20-75) \mathrm{u} / \mathrm{l}$ & Urine culture & No Growth \\
\hline Lipase & $29 u / l(4-24) u / l$ & Blood culture & No Growth \\
\hline ESR & $61 \mathrm{~mm} / \mathrm{hr}(0-10) \mathrm{mm} / \mathrm{hr}$ & UA & Large Ketones \\
\hline
\end{tabular}

Values in parenthesis indicate normal range. PT_-Prothrombin time; INR_-International normalized ration; PTT—Partial thromboplastin time; AST —Aspartate aminotransferase; ALT_Alanine aminotransferase; Alk Phos-Alkaline phosphatase; ESR_Erythrocyte sedimentation rate; HBsAG_Hepatitis B surface antigen; Anti-HBs-Hepatitis B surface antibody; Anti-HBc-Hepatitis B core antibody, total; IgM-HBc-Hepatitis B core immunoglobulin M antibody; Anti-HCV-Hepatitis C core virus antibody; HIV ELISA-Human immunodeficiency virus enzyme-linked immunosorbent assay; C3/4—Complement 3 and 4; ANA—Antinuclear antibodies; RF- Rheumatoid factor; Anti-SMA—Anti-smooth muscle antibody; TSH— Thyroid stimulating hormone; CEA-Carcinoembryonic antigen; UA-Urinalysis.

clinical response and resolution of ascites. However, further studies of this correlation are needed. Additionally, Adenosine Deaminase (ADA) an aminohydrolase that converts adenosine to inosine, has proven to be a helpful diagnostic tool. An ascitic ADA activity of $>30 \mathrm{U} / \mathrm{L}$, in the absence of immunosuppression or cirrhosis, has a sensitivity of $96 \%$ and specificity of $98 \%$ in diagnosis of TbP [9]. ADA was elevated in the index case further adding to the suspicion of TbP.

In addition to biochemical analysis, radiographic and morphologic studies are performed to elucidate a definitive diagnosis. Computer Tomography (CT) of the abdomen is the most useful radiographic study. CT findings in TbP include peritoneal thickening, lymphadenopathy, omental caking and the presence of ascites with fine mobile septations [11]. Whereas, abdominal CT is useful, the laparoscopic surgical approach remains the ultimate means to confirm the diagnosis of TbP. Not only does it allow for inspection of the peritoneum but also offers the option of obtaining biopsy specimen. Moreover, this diagnostic approach showed impressive sensitivity and specificity rates of $93 \%$ and $98 \%$ respectively, when the macroscopic appearance is combined with histological findings [10]. The typical macroscopic appearance of infected peritoneum includes regular white granules of the omentum that are numerous, dispersed and friable on biopsy, hyperemia of the peritoneum, filamentous peritoneal adhesions and clumping of bowel loops with fibrin deposition. Histologically, typical lesion are caseating epithelioid granuloma containing multi nucleated giant cells, epithelioid hystiocytes and lymphocytes forming a ring surrounding a central area of caseating necrosis [9]. In the case presented, the macroscopic appearance was consistent with the typical findings. However, the histologic findings were unusual and contrasted with typical findings, showing granulomatous inflammation without characteristic caseating necrosis (Figure 2). Some patients may have non caseating granulomas, an indication that necrosis has not manifested. Caseation usually occurs in about 15 - 30 days. However, it is also possible that more diverse sections could reveal caseation [5].

The treatment of TbP is medical. The delay in initiating treatment may increase mortality. The overall mortality in one study was 35\% [7]. The current recommended treatment protocol combines four drugs, Isoniazid (INH), Rifampin (RIF), Pyrazinamide (PZA) and Ethambutol (EMB) given for 2 months, relayed by 4 months 
of combination therapy of INH and RIF for a total of 6 months but may be extended to 9 months. The favorable response to treatment results in the resolution of symptoms and the disappearance of ascites. Laboratory abnormalities will normalize within 3 months after starting treatment [9].

\section{Conclusion}

Clinicians in developed countries need to have a high clinical index of suspicion of TbP, especially when patients were present with vague symptoms such as ascites and the typical demographics. Laparoscopic biopsy is the most reliable, safe and quick method for the diagnosis of TbP with the typical pathologic biopsy finding of caseating granuloma. However, the absence of caseating granuloma does not exclude diagnosis. The clinic data must be assessed in its entirety because missing the diagnosis will lead to increased morbidity and mortality.

\section{Disclosure Statement}

The Authors have nothing to disclose.

\section{References}

[1] Yeh, H.F., Chiu, T.F., Chen, J.C. and Ng, C.J. (2012) Tuberculous Peritonitis: Analysis of 211 Cases in Taiwan. Digestive and Liver Disease, 44, 111-117. http://dx.doi.org/10.1016/j.dld.2011.08.020

[2] Khan, F.Y., Al-Muzrakchi, A.M., Elbedawi, M.M., Al-Muzrakchi, A.A. and Al Tabeb, A. (2012) Peritoneal Tuberculosis in Qatar: A Five-Year Hospital-Based Study from 2005 to 2009. Travel Medicine and Infectious Disease, 10, 2531. http://dx.doi.org/10.1016/j.tmaid.2011.12.001

[3] Demir, K., Okten, A., Kaymakoglu, S., Dincer, D., Besisik, F., Cevikbas, U., et al. (2001) Tuberculous PeritonitisReports of 26 Cases, Detailing Diagnostic and Therapeutic Problems. European Journal of Gastroenterology \& Hepatology, 13, 581-585. http://dx.doi.org/10.1097/00042737-200105000-00019

[4] Lee, W.K., Van Tonder, F., Tartaglia, C.J., Dagia, C., Cazzato, R.L., Duddalwar, V.A., et al. (2012) CT Appearances of Abdominal Tuberculosis. Clinical Radiology, 67, 596-604. http://dx.doi.org/10.1016/j.crad.2011.11.003

[5] Fedotin, M.S. and Brewer, D.L. (1972) Noncaseating Tuberculous Peritonitis. Archives of Internal Medicine, 130, 920-922. http://dx.doi.org/10.1001/archinte.1972.03650060110020

[6] Vardareli, E., Kebapci, M., Saricam, T., Pasaoglu, O. and Acikalin, M. (2004) Tuberculous Peritonitis of the Wet Ascitic Type: Clinical Features and Diagnostic Value of Image-Guided Peritoneal Biopsy. Digestive and Liver Disease, 36, 199-204. http://dx.doi.org/10.1016/j.dld.2003.10.016

[7] Marshall, J.B. (1993) Tuberculosis of the Gastrointestinal Tract and Peritoneum. The American Journal of Gastroenterology, 88, 989-999.

[8] Manohar, A., Simjee, A.E., Haffejee, A.A. and Pettengell, K.E. (1990) Symptoms and Investigative Findings in 145 Patients with Tuberculous Peritonitis Diagnosed by Peritoneoscopy and Biopsy over a Five-Year Period. Gut, 31, 1130-1132. http://dx.doi.org/10.1136/gut.31.10.1130

[9] Guirat, A., Koubaa, M., Mzali, R., Abid, B., Ellouz, S., Affes, N., et al. (2011) Peritoneal Tuberculosis. Clinics and Research in Hepatology and Gastroenterology, 35, 60-69. http://dx.doi.org/10.1016/j.gcb.2010.07.023

[10] Sanai, F.M. and Bzeizi, K.I. (2005) Systematic Review: Tuberculous Peritonitis-Presenting Features, Diagnostic Strategies and Treatment. Alimentary Pharmacology \& Therapeutics, 22, 685-700. http://dx.doi.org/10.1111/j.1365-2036.2005.02645.x

[11] Akhan, O. and Pringot, J. (2002) Imaging of Abdominal Tuberculosis. European Radiology, 12, 312-323. http://dx.doi.org/10.1007/s003300100994 


\section{Abbreviations}

ADA: Adenosine deaminase

AIDS: Acquired immune deficiency syndrome

Alk Phos: Alkaline phosphatase

ALT: Alanine aminotransferase

ANA: Anti nuclear antibody

Anti-HBc: Hepatitis B core antibody

Anti-HBs: Hepatitis B surface antibody

Anti-HCV: Hepatitis C core virus antibody

Anti-SMA: Anti-smooth muscle antibody

AST: Aspartate aminotransferase

C3/4: Complement 3 and 4

CEA: Carcinoembryonic antigen

CT: Computer Tomography

ECA: Emergency care area

EMB: Ethambutol

ESR: Erythrocyte sedimentation rate

HBsAG: Hepatitis B surface antigen

HIV ELISA: Human immunodeficiency virus Enzyme-linked immunosorbent assay

IgM-HBc: Hepatitis B core immunoglobulin M antibody

INH: Isoniazid

INR: International normalized ration

LDH: Lactate Dehydrogenase

PPD: Purified protein derivative

PT: Prothrombin time

PTT: Partial thromboplastin time

PZA: Pyrazinamide

RBC: Red blood cell count

RF: Rheumatoid Factor

RIF: Rifampin

SAAG: Serum to ascetic albumin gradient

TbP: Peritoneal tuberculosis

TSH: Thyroid stimulating hormone

UA: Urinalysis

WBC: White blood cell count 